\title{
Problemes aux limites en theorie des distributions.
}

\author{
Paul Krige (*)
}

Résumé. - Etude de la méthode des potentiels pour résoudre des problèmes aux limites relatifs $\grave{a}$ certains opèrateurs différentiels. Résolution et discussion des problèmes aux limites pour un système diffèrentiel elliptique lorsque les données sont des distributions (le problème étant elliptique d̀ droite ou $\grave{a}$ gauche).

Soit $\bar{\Omega}$ une variété compacte $\left(\mathfrak{C}^{\infty}\right.$ et réelle) de bord $\omega$ plongée dans une variété $\mathfrak{C}^{\infty}$ et réelle $M, \overline{\mathbf{Q}}$ et $M$ ont la mème dimension $n . \stackrel{P}{P}$ désigne un opérateur différentiel elliptique d'ordre $m$.

$$
\mathfrak{C}^{\infty}(M, E) \rightarrow \mathfrak{C}^{\infty}(M, F)
$$

où $E$ et $F$ sont deux fibrés vectoriels complexes de base $M . B$ désigne un opérateur pseudo différentiel (o.p.d.) sur $\omega$ :

$$
\mathcal{C}^{\times}\left(\omega, E^{m}\right) \rightarrow \mathfrak{C}^{\infty}(\omega, G)
$$

où $G$ est un fibré vectoriel complexe de base $\omega$ et $E^{m}$ est la somme directe de $m$ exemplaires du fibré $E$. Soit $v_{j}$ dans $\mathfrak{C}^{\infty}(\omega, E), j=0,1 \ldots m-1$. Soit $k$ le plus petit entier tel que $B\left(v_{0}, \ldots, v_{m-1}\right)$ ne dépende que de $\left(v_{0}, \ldots v_{k-1}\right)$ seulement. $V$ étant un champ de vecteurs defini dans $M$ au roisinage de $\omega$ et transverse à $\omega$, on suppose qn'une carte de $M$ transforme localement $M$ en $R_{x}^{n}, \boldsymbol{Q}$ en le demi-espace où $x_{n}>0$ et $V$ en $\frac{\partial}{\partial x_{n}}$. On se donne $f$ dans $\mathfrak{D}^{\prime}(\boldsymbol{\Omega}, F)$ et $g$ dans $\mathfrak{D}^{\prime}(\omega, G)$. Le problème aux limites général relatif à ces données consisterait à:

trouver $u$ dans $\mathfrak{D}^{\prime}(\Omega, F)$ tel que

$$
\begin{gathered}
P u=f \text { dans } Q \\
B(\gamma u)=g \text { sur } \partial \mathbf{Q}=\omega
\end{gathered}
$$

où $\gamma u$ est formé par la collection des traces transverses de $u$ sur le bord $\omega$ (ò̀ $\left.x_{n}=0\right) u_{j}=\gamma_{j} u=\left(\frac{1}{i} \frac{\partial}{\partial x_{n}}\right)^{j} u(\cdot, 0)$ avec $j=0,1 \ldots, m-1$. Le problème

(*) Entrata in Redazione il 6 dicembre 1968 . 
de Dirichlet est un problème de ce type avec $M=\boldsymbol{E}_{x}^{n}, \mathbf{Q}=$ boule unité, $P=\Delta, m=2, k=1$. Pour le problème de Neuman, on a $k=2$ et $X$ est le champ des vecteurs normaux...

Lorsque les traces $u_{j}(j=0, \ldots k-1)$ sont « naturellement définies» (par exemple lorsque $u$ est dans $\mathcal{C}^{4}(\overline{\mathbf{Q}})$ ), l'interprétation du problème (1) ne présente aucune difficulté: on dira que le problème (1) a naturellement un sens.

Mais lorsqu'il n'en est plus ainsi (par exemple lorsque $u$ est Coc dans $Q$ et à croissance lente au voisinage du bord), il est nécessaire de préciser le sens dans lequel le problème (1) doit être interprété. Il nous faut donc donner un sens au problème (1) en théorie des distributions. On peut se poser des problèmes analogues d'interprétation pour des opérateurs $P$ non elliptiques.

Dans le cas où $P$ est une équation, où $B$ est un système normal d'opérateurs différentiels sur le bord qui recouvre $P$, et où $f$ satisfait à certaines restrictions, Lions et Magenès ([14]) ont interprété le problème (1) à l'aide d'ane formule de GrEEN; ces auteurs ont aussi discuté (1) en utilisant des techniques de transposition et d'interpolation. Dans le cas où $P$ est un o.p.d. de type 0 , une interprétation et une résolution de (1) peuvent être déduites du travail [4] de L. Bovtet de Monvel les traces étant interprétées à l'aide de noyaux de Poisson ([3]).

Dans le présent travail, on se fixe à priori deux interprétations naturelles et directes des $\gamma \boldsymbol{u}(j=0, \ldots k-1)$ :

- ou bien comme valeurs au bord ( $u$ étant semi-régulière par rapport à $x_{n}$ : voir [18])

- ou bien comme traces fonctionnelles (définies par prolongement continu).

Puis l'on énonce une condition nécessaire et suffisante pour que le probleme (1) ait un sens relativement à ces interprétations: voir $\& 4$

(2) On fait l'hypothèse essentielle que la distribution u cherchée se prolonge en une distribution définie sur $M$ : l'exemple (6.c) montre qu' une hypothèse de ce genre est nécessaire si l'on ne veut pas utiliser des fonctions généra. lisées qui ne soient pas des distributions.

On énonce (\$ 3) deux résultats prouvant l'existence de valeurs an bord (voir definition an $\$ 1$ ) pour ne solution $u$ de l'équation homogène. Le deuxième résultat vaut même si $P$ est non elliptique et permettrait de généraliser les classes da HARDY $H^{P}$. On indique aussi une généralisation de la formule de GreEN (dans le cas de solutions distributions) et un théorème de prolongement des solutions à travers une hypersurface régulière. On étudie au $\$ 2$ la représentation d'une solution de l'equation homogène à l'aide de potentiels. Vus ces préliminaires, on constate alor's (\$ 5) que la méthode de 
L. Hörmander ([12]) pour résondre (resp. discuter) un problème du type (1) elliptique à droite (resp. à gauche) dans le cas où $\gamma u$ a directement un sens, s' etend au cas où la trace $\gamma u$ doit être interprètée. Notons que la méthode ci-dessus permet de résoudre (resp. discuter) les problèmes (1) elliptiques à droite (resp. à gauche) dans des espaces de distributions différents des espaces de Sobolev $H^{s}$. (par exemple des espaces $H^{s}$ anisotropes, des espaces de Besov $B_{q}^{s, p}$, et en particulier des espaces de fonctions hölderiennes). En effet, alors que la méthode de Lions Magenis nécessite entre autres des propriétés de transposition et d'interpolation (voir problème 11.3 et 11.4 de [14], la méthode employée ici et détaillée au point (37) pour les espaces de Sobolev, utilise simplement des propriétés de prolongement, de trace, et de continuité de l'opérateur associé à un o.p.d.

\section{\& 1. - Preliminaires.}

Nous rappelons d'abord quelques résultats sur les distributions prolon. geables (pour résoudre (1), on supposera tonjours que $u$ est une distribution prolongeable). Afin de définir les deux interprétations da problème (1) que nous considèrerons ( $\$ 4$ ) nous définissons ci-après les valeurs aa bord transverses ef les traces fonctionnelles d'une distribution (non quelconque) sur $\Omega$.

Distributions prolongeables.

Soit $\Omega$ un ouvert de $\boldsymbol{R}^{n}$. On peut, dans ce qui suit, remplacer $\boldsymbol{R}^{n}$ par une variêté réelle dénombrable à l'infini $M$. Soit $T$ dans $\mathfrak{D}^{\prime}(\mathbf{Q}$ ). (de même, on peut considérer des sections distributions de fibrés au lieu de distributions à valeurs complexes). On dit que $T$ est prolongeable s' il existe $\tilde{T}$ dans $\mathscr{D}^{\prime}\left(\boldsymbol{R}^{n}\right)$, telle que $T$ soit la restriction de $\tilde{T}$ à $\mathcal{C}_{0}^{\infty}(\mathbf{Q})$. L'ensemble des distributions, définies sur $\Omega$ et qui sont prolongeables, est noté $\mathfrak{D}_{p}^{\prime}(\Omega)$.

\section{(3) Proposition.}

a) Pour que la distribution $T$ définie sur $\Omega$ soit prolongeable, il faut el il suffit que la forme linéaire $T$ sur $\mathfrak{C}_{0}^{\infty}(\Omega)$ soit continue, lorsque $\mathfrak{C}_{0}^{\infty}(\mathbf{Q})$ est muni de la topologie induite par $\mathcal{C}_{0}^{\infty}\left(\boldsymbol{R}^{n}\right)$.

b) Si $T$ est une distribution prolongeable définie sur $\Omega$, il existe un prolongenent de $T$ porté par $\bar{\Omega}$.

Cette proposition résulte du théorème de HaHN Banach. Notons que la condition $(3-a)$ peut s'écrire:

$$
\begin{gathered}
\forall K \Subset R^{n}, \quad \exists C \text { et } m \text { t.q. }: \forall \varphi \in \mathcal{C}_{0}^{\infty}(\Omega), \quad \text { Supp } \varphi \subseteq K ; \\
|<T, \varphi>| \leq C \sup _{\substack{x \in K \\
\alpha \mid \leqslant m}}\left|\left(\frac{\partial}{\partial x}\right)^{x} \varphi(x)\right|
\end{gathered}
$$


On a la:

(5) Caractérisation des distributions prolongeables.

$\mathfrak{D}\left(R^{n}, \mathbb{Q}^{\mathrm{Q}}\right)$ désigne l'ensemble des fonctions de $\mathfrak{D}\left(\boldsymbol{R}^{n}\right)$ qui s' annulent sur $\mathbf{Q}^{\mathrm{Q}}$ ainsi que toutes leurs dérivées. Cet espace est muni de la topologie de $\mathscr{D}\left(\boldsymbol{R}^{n}\right)$. Alors l'ensemble des distributions prolongeables définies sur $\mathrm{\Omega}$ s'identifie au dual topologique de $\mathfrak{D}\left(R^{n}\right.$, [D).

(6) Exemples. de $\Omega$ :

a) Soit $f$ mesurable: $\Omega \rightarrow C$, à croissance lente au voisinage du bord

$$
\forall K \Subset \boldsymbol{R}^{n}, \quad \exists K>0 \quad \text { t.q. } \quad \forall x \in K \cap \Omega, \quad f(x)=\mathcal{O}\left(d^{k}(x, Q \Omega)\right)
$$

On suppose que $\Omega$ est un «ouvert régulier» (voir (7)).

Alors, on peut définir un prolongement de $r$ par un procédé de partie finie en utilisant une partie finie réelle ou complexe (voir [17]).

b) Toute distribution prolongeable représentée par une fonction $\mathfrak{C}^{\infty}$ n'est pas nécessairement à croissance lente an voisinage du bord. Si l'on considère par exemple la distribution $T$ sur $R$ représentée par la fonction

$$
T(x)=\left\{\begin{array}{lll}
0 & \text { si } \quad x<0 \\
\sin \left(\exp \frac{1}{x}\right) & \text { si } x>0
\end{array}\right.
$$

$T$ est portée par $\underline{Q}=\boldsymbol{R}^{+}$. Donc, la restriction de $f=T^{\prime}$ à $\boldsymbol{R}^{+}$est prolongeable, et pourtant, $f$ n'est pas à croissance lente au voisinage de l'origine.

c) Si $f$ est solution dans un ouvert régulier $\mathbf{Q} d$ ' un opérateur différentiel elliptique $P, f$ n'est pas forcément prolongeable en une distribution définie sur $\boldsymbol{R}^{n}$. Par exemple, prenons:

$$
n=2, \quad P=\frac{\partial}{\partial z}, \quad \Omega=\text { le disque unité, } \quad f(z)=\exp \left(\frac{1}{1-z}\right) .
$$

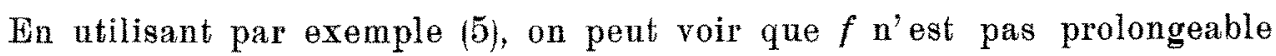
en une distribution.

(7) Ouvert régulier $\Omega$, surfaces parallèles $\omega_{\varepsilon}$, dérivées transverses.

On suppose que $\overline{\boldsymbol{\Omega}}$ est une variété $C^{\circ}$ à bord de la variété $M$, dénombrable à l'infini. $(\overline{\mathrm{Q}}$ et $M$ étant de même dimension $n)$. On se donne un champ de vecteur $V$ defini sur $M$ au voisinage du bord $\omega$ de $\bar{\Omega}$, transverse à $\omega$ et 
dirigé vers l'intérieur de $\mathbf{Q}$. On peut résoudre localement (et pour $t$ petit) le système différentiel $\frac{d x}{d t}=X, x(0)=x_{0} \in \omega$ ce qui permet localement dans $\omega$ :

- de définir les lignes de force du champ $X$

- de définir des coordonnées ourvilignes dans $M$ au voisinage de $\omega$, le point $x(t)$ étant repéré par $\left(x_{0}, t\right)$.

Si $\omega$ est compact, ces coordonnées sont définies pour $|\emptyset|<\varepsilon_{0}$. On peut done supposer qu'au voisinage de $\omega$, la structure de variété de $M$ est définie par un nombre fini de cartes ayant la propriété suivante. Pour $x$ queleonque dans $\omega$, il existe un voisinage de $x$ dans $\bar{\Omega}$ transformé en un voisinage de l'origine dans le demi-plan de $\boldsymbol{R}_{x}^{n}$ où $x_{n}>0$. Le bord $\omega$ est transformé localement en l' hyperplan où $x_{n}=0$, le champ $V$ étant appliqué sur le champ $\frac{\partial}{\partial x_{n}}$.

Pour simplifier, le champ $V$ est noté $\frac{\partial}{\partial x_{n}}$. On appelle bord parallèle $\left.\omega_{\varepsilon}|| \varepsilon \mid<\varepsilon_{0}\right)$ la sous-variété de $M$ où $t=\varepsilon$. Chaque carte définie cidessus applique localement $\omega_{\varepsilon}$ sur l'hyperplan de $\boldsymbol{R}_{x}^{n}$ où $x_{n}=\varepsilon$.

Dans le cas où le bord $\omega$ n' est pas compact, il n' est pas certain qu'il existe un champ transverse $X$ tel que pour $\varepsilon_{0}$ suffisamment petit, les cartes locales définies ci-dessus existent pour tout $|t| \leq \varepsilon_{0}$ au voisinage de tout $\omega$. Nous limiterons au cas où an tel champ $X$ existe.

(8) Valeurs au bord et traces.

Pour simplifier, on lit toute distribution sur $M$ définie au voisinage de $\omega$ à l'aide des cartes définies précédemment.

(9) Définition.

Soit $\mathbf{Q}$ une variété à bord et $u$ dans $\mathfrak{D}^{\prime}(\mathbf{Q})$.

$k$ si

a) On dit que $u$ admet des valeurs au bord transverse jusqu'à l'ordre

$$
\left(\frac{1}{i} \frac{\partial}{\partial x_{n}}\right)^{\mu} u\left(x^{\prime}, x_{n}\right) \in \mathfrak{C}^{0}\left(\boldsymbol{R}_{x_{i n}}^{+}, \mathfrak{D}^{\prime}\left(\left(\boldsymbol{R}_{x^{\prime}}^{n-1}\right)\right) \quad \mu=1, \ldots k\right.
$$

c'est-à-dire si $u\left(x^{\prime}, x_{n}\right) \in \mathfrak{C}^{k}\left(\boldsymbol{R}_{x_{n}}^{+}, \mathfrak{D}^{\prime}\left(\boldsymbol{R}_{x}^{n-1}\right)\right)$

on pose alors

$$
y^{\mu} u=\left(\frac{1}{i} \frac{\partial}{\partial x_{n}}\right)^{\mu} u(\cdot, 0)
$$

$\gamma_{\mu} u$ est appelée la trace transverse d'ordre $\mu$. 
b) Soit $X$ un sous espace vectoriel topologique de $\mathfrak{D}_{P}^{\prime}(\Omega)$ où $X \cap \mathcal{C}^{\infty}(\overline{\Omega)}$ est dense. On dit que les éléments de $X$ admetlent des traces transverses jusqu'd $l$ 'ordre $k$ si les applications (pour $0 \leq \mu \leq k$ ):

$$
\begin{gathered}
\mathcal{C}^{\infty}(\bar{\Omega}) \cap X \rightarrow \mathcal{C}^{\infty}(\omega) \\
u \rightarrow \gamma_{\mu} u
\end{gathered}
$$

se prolongent par continuité en des applications continues de $X$ dans $\mathfrak{D}^{\prime}(\omega) . \gamma_{\mu}$ est appelé la $X$-trace trasverse d'ordre $\mu$ de $u$.

(10) Prolongement de distributions admettant des valeurs au bord transverse, jusqu' à l'ordre $k$.

En atilisant par exemple le développement de TAYLOR à l'origine da la fonction $x_{n} \rightarrow u\left(\cdot, x_{n}\right)$, on voit que toute $u$ de $\mathcal{C}^{k}\left(\boldsymbol{R}_{x_{n}}^{+}, \mathscr{D}^{\prime}\left(\boldsymbol{R}_{x^{\prime}}^{n-1}\right)\right)$ admet un prolongement dans $\mathfrak{C}^{4}\left(\boldsymbol{R}_{x_{n}}, \mathfrak{D}^{\prime}\left(\boldsymbol{R}_{x^{\prime}}^{n-1}\right)\right)$ done dans $\mathscr{D}^{\prime}\left(\boldsymbol{R}_{x_{n}}, \mathfrak{D}^{\prime}\left(\boldsymbol{R}_{x^{\prime}}^{n-1}\right)=\mathfrak{D}^{\prime}(M)\right.$.

(11) Si une distribution admet une valeur au bord d'ordre 0, elle est prolongeable. En prolongeant la fonction $x_{n} \rightarrow u\left(\cdot, x_{n}\right)$ par 0 pour $x_{n}<0$, on obtient une fonction bornée à valeurs dans $\mathscr{D}^{\prime}\left(\boldsymbol{R}_{x_{n}}, \mathfrak{D}^{\prime}\left(\boldsymbol{R}_{x^{\prime}}^{n-1}\right)\right.$ c' est-à-dire une distribution sur $\boldsymbol{R}^{n}$ : cette distribution est appelée le prolongement canonique de $u$.

Noter qu' on peut considérer $\gamma_{\mu} u(1 \leq \mu \leq k)$ comme la valeurs au bord transverse d'ordre $\mu$ d'un prolongement de $u$.

De la même manière, avec les hypothèses de $(9-b)$, s'il existe un sousespace vectoriel topologique $\tilde{X}$ de $\mathfrak{D}^{\prime}(M)$ tel que

- l'application de restriction $\grave{a} \Omega: \tilde{X}$ restr. $>X$ soit surjective

- cette application admet un relevement continu rel: $X \rightarrow \tilde{X}$, alors on peut considérer $\gamma_{\mu} \imath$ comme la $\tilde{X}$-trace transverse d'ordre $\mu$ d'un relèvement $\tilde{u}$ de $u$.

Exemples

a) Espaces $H^{P}$ de Stein et Weiss.

$$
\mathbf{Q}=\boldsymbol{R}_{x}^{n+}=\boldsymbol{R}_{x_{n}}^{+} \times \mathbb{R}_{x^{\prime}}^{n-1}, \quad \text { et } P(x, D)=\Delta .
$$

Soit $u$ une fonction définie et harmonique sur $\Omega$ et telle que

$$
u \in L^{\infty}\left(\boldsymbol{R}_{x_{n}}^{+}, L^{P}\left(\boldsymbol{R}_{x^{\prime}}^{n-1}\right)\right) \quad \text { soit } \sup _{x_{n}>0} \int\left|f\left(x^{\prime}, x_{n}\right)\right|^{p} d x^{\prime}<\infty
$$


$u$ appartient $\grave{a} \mathfrak{D}_{P}^{\prime}(\mathrm{Q})$. On peut montrer que $u$ admet une valeur au bord dans $L^{P}$ (voir [18] et aussi $(28-a)$ ) si $1<p<\infty$. La classe de telles fonctions $u$ généralise la classe $H^{P}$ de Hardx des fonctions holomorphes pour $|z| \leq 1$ et telles que

$$
\operatorname{snp}_{r<1} \int_{0}^{2 \pi} \mid f\left(\mathrm{r} \theta^{i \theta} d \theta<\infty\right.
$$

b) Espaces $H^{s}$ de Sobolev.

Les théorèmes usuels (voir [7] chap. 2) permettent de montrer que toute distribution $u$ de $H_{\text {loc }}^{s}$ admet des valeurs au bord transverses d'ordre $k$ si $s>k+\frac{1}{2}$.

c) Espaces $H^{s}$ avec poids.

On pose

$$
H_{a}^{s}\left(\boldsymbol{R}_{+}^{n}\right)=\left\{u \in \mathscr{D}^{\prime}\left(\boldsymbol{R}_{+}^{n}\right) ; \quad x_{n}^{\alpha} D^{\beta} u \in L^{2}\left(\boldsymbol{R}_{+}^{n}\right) \quad \text { pour }|\beta| \leq s\right\}
$$

De [6], il résulte que les distributions de cet espace admettent des $H_{\alpha}^{s}$ - traces fonctionnelles si $s>\alpha+\frac{1}{2}$ et que

- ces traces sont nulles si $\alpha+\frac{1}{2} \leq 0$

- ces traces sont dans $H^{s-\alpha-} \frac{1}{2}$

Noter que si $\alpha<0$, les éléments de $H_{\alpha}^{s}\left(\boldsymbol{R}_{+}^{n}\right) \mathbf{n}^{\prime}$ ont pas de traces (pour $s$ convenable) sur un hyperplan où $x_{n}$ est une constante positive.

\section{§ 2. - Representation d'une solution de l'equation homogene a l'aide} de potentiels.

Soit $\overline{\mathbf{\Omega}}$ une varióté à bord $\mathcal{C}^{\infty}$ de la variété $\mathcal{C}^{\infty}$ réelle $M$ (dim $\overline{\mathbf{Q}}=$ $=\operatorname{dim} M=n$ ). Soit $P$ un système différentiel d'ordre $m$ sur $M$. (on étudie au voisinage du bord $\omega$ de $\bar{\Omega}$ les cartes définies au $\S$ précédent. Si $A$ est une distribution sur $M$ supportée par $\omega$, elle s'écrit localement

$$
A=\sum_{\nu=0}^{M} u_{\nu}\left(x^{\prime}\right) \times\left(\frac{\partial}{\partial \dot{\partial} x_{n}}\right)^{\nu} \varepsilon_{0}\left(x_{n}\right)
$$

avec $\boldsymbol{u}_{\nu}$ dans $\mathcal{G}^{\prime}\left(\boldsymbol{R}_{x^{\prime}}^{n-1}\right)$. On dit que $u_{\nu}$ est la densité de la multicouche $\boldsymbol{u}_{\nu} \times \delta(v)$. 
Soit $T$ un paramétrise à ganche de $P$ :

$$
T \circ P=I d+r
$$

où $r$ est un opérateur de noyau $\mathfrak{C}^{\infty}$. On suppose que $T$ envoie $\mathfrak{D}^{\prime}(M)$ dans $\mathfrak{D}^{\prime}(M) . T(A)$ est appelé le potentiel de la distribution $A$. Si $u$ dans $\mathfrak{D}_{P}^{\prime}(\Omega)$ est telle que $P u=0$, on cherche à représenter $u$ (dans $\Omega)$ comme un potentiel (d'une charge étalée sur w). La proposition (15) donne des cas où une telle représentation existe à une fonction $\mathcal{C} \times$ près. On cherche ensuite à simplifier cette représentation. Puis l'on montre que si $P$ est elliptique, $u$ est à crois. sance lente au voisinage du bord (l'intérèt de ceci résulte de (6.b). Si $v$ est dans $\mathfrak{C}^{0}(\Omega), v^{0}$ désigne la distribution sur $M$ représentée par la fonction égale à $v(x)$ pour $x$ dans $\Omega$ et par 0 si non.

Proposition.

a) Soit $u$ dans $e^{m}(\overline{\mathbf{\Omega}})$.

(15') Alors $P\left(u^{0}\right)=(P u)^{0}+\tilde{P}(\gamma u)$ oì $\tilde{P}(\gamma u)$ est une distribution étalée sur วQ et qui dépend de $u$, seulement par l'intermédiaire de la donnée de Canchy:

$$
\gamma u=\left(\gamma_{0} u, \gamma_{1} u, \ldots \gamma_{m-1} u\right)
$$

Si $P u=0$ dans $Q$, on $a$

$$
u^{0}+r u^{0}=T(\tilde{P}(\gamma u))
$$

b) Soil $u$ dans $\mathfrak{D}_{p}^{\prime}(\mathbf{Q})$ telle que $P u=0$.

Soit $\tilde{u}$ un prolongement de u porté par $\overline{\mathbf{g}}$. Alors $P \tilde{u}=A$, A étant une distribution porlée par $\omega$ et

$$
T P \tilde{u}=\tilde{u}+\tilde{u}=T A .
$$

Les iormules (16) et (17) figurent dans [10], si $P$ est elliptique.

(18) Remarque sur la représentation sous forme de potentiel d'une solution $u$ de l'équation homogène $P u=0$.

Pour simplifier, on suppose que $T$ est une solution élémentaire bilatère de $P .(T P=I d, P T=I d)$ et que $u$ est dans $\mathcal{C}(\bar{Q})$. On peut se poser les;

$$
\text { questions }
$$

telles que;

a) Que peut-on dire de toutes les distributions $A$ de support $\omega$ et

$$
u=T A \text { dans } Q .
$$


b) Comment peut-on réduire le nombre de multicouches composant $A$ si $A$ est telle que (20).

Notons que les traces $\gamma_{j} u(j=0, \ldots m-1)$ sont reliées par des o.p.d.: voir par exemple [10]. De plus, L. Bovtet de Monvel a remarqué (voir [4]) que si le bord est non caractéristique pour $P$, il existe $A$ vérifiant (20), $A$ étant composée par des multiconches d'ordre au plus $m-1$. On pent noter encore ceci.

\section{(21) proposition.}

Soit A' une distribution portée par w formée par une somme de multicouches de densité ${ }^{\infty}$. Pour que $A^{\prime}$ soit telle que $T A^{\prime}=0$ dans $\Omega$, il faut et il suffit $q u^{\prime}$ il existe $v$ dans $\mathrm{C}^{\times}\left(\mathrm{C}^{\Omega}\right)$ telle $q u e A^{\prime}=\bar{P}^{\prime}(\gamma v)$ et $P v=0$ dans $\mathrm{Q} \mathrm{Q}$.

Cette proposition est vraie sous les:

\section{Conditions}

(a) Si ${ }^{0} v$ est la distribution sur $M$ représentée par la fonction $v(x)$ sur $Q^{Q}$ et par 0 sur $\Omega, \tilde{P}^{\prime}(\gamma v)$ est défini par la formule de GREen

$$
P\left({ }^{0} v\right)={ }^{0}(\tilde{P} v)+\tilde{P}^{\prime}(\gamma v)=\tilde{P}^{\prime}(\gamma v)
$$

(b) $T$ agit sur $\tilde{P}^{\prime}(\gamma v)$

(c) $T$ vérifie la condition de la régularite: $T A^{\prime}$ est dans $\mathcal{C}^{\infty}\left(\mathcal{C}^{\mathrm{Q}}\right)$ si les densités de $A^{\prime}$ sont $\mathcal{C}^{\infty}$.

En effet:

La condition de (21) est suffisante car vu (22.a) et (22.b), on a

$$
T P\left({ }^{0} v\right)={ }^{0} v=T \tilde{P}^{\prime}(\gamma v)
$$

donc, le potentiel de $\tilde{P}^{\prime}(\gamma v)$ est nul sur $\Omega$. Réciproquement si $T A^{\prime}=0$ dans $\mathrm{Q}, v=T A^{\prime}$ est porté par $\mathrm{Q}^{\mathrm{Q}}$ et vu (22.c), $v$ est dans $\mathcal{C}^{\infty}\left(\mathrm{C}^{\mathrm{Q}}\right)$.

On a $P v=P T A^{\prime}=A^{\prime}$. Vu $(22 . a)$, on a $A^{\prime}=\tilde{P}^{\prime}(\gamma v)$.

\section{Exemple.}

Soit $u$ harmonique dans un domaine $\Omega$ de $M=E^{n}$, et $u$ dans $\mathcal{C}^{\infty}(\bar{\Omega}), \Delta$ admet une solution élémentaire bilatère $T$. On prend pour $V$ un champ des vecteurs normaux a la frontière $\omega$ de $Q$. $V u(16)$, on a $u=T \tilde{P}(\gamma u)$ avec $\tilde{P}(\gamma u)=u_{0} \times \delta^{\prime}+u_{1} \times \delta$. Si l'on sait trouver $v$ dans $\mathcal{C}^{\infty}(\mathcal{C}$ ), telle que $\Delta v=0$ dans $\mathcal{Q}^{\bar{Q}}$, admettant $u_{1}$ comme trace d'ordre 1 , alors $u=T\left(\tilde{P}(\gamma u)+A^{\prime}\right)$ dans $\mathbf{Q}$ avec $A^{\prime}=-u_{1} \times \delta+\ldots$ 
Donc $u=T A$ dans $\Omega$ où $A$ est une double couche étalée sur $\partial \Omega$. C'est cette représentation qui est utilisée pour résoudre le théorème de Dirichlet. On peat montrer de la même façon que toute fonction harmonique dans un ouvert régulier de $\boldsymbol{R}^{n}$ pent ètre représentée comme le potentiel d'une simple couche.

Relation entre la régularité des charges el celle de leurs potentiels.

On sait (Cf [3], [11], [17]) que si $T$ est un paramétrix d'un opérateur différentiel elliptique, $T$ vérifie la condition de la régularité. On a encore la

$$
\text { proposilion. }
$$

Soit $T$ un opérateur pseudo différentiel et: $A=u\left(x^{\prime}\right) \times \delta_{0}^{(x)}\left(x_{n}\right)$ avec $u$ dans $\mathscr{B}^{\prime}$. Alors le potentiel $T A$ est $\grave{a}$ croissance lente au voisinage du bord.

Démonstration.

Quitte à remplacer $T$ par $T \circ\left(D_{n}\right)^{\mu}$, on peut supposer que $\mu=0$. Soit $p(x, x-y)$ le noyau de $T$. On a $v=T A$ avec

$$
\begin{aligned}
v(x) & =\iint p(x, x-z) u\left(z^{\prime}\right) \times \delta_{0}\left(z_{n}\right) d z^{\prime} d z_{n} \\
& =\int p\left(x^{\prime}, x_{n} ; x^{\prime}-z^{\prime}, x_{n}\right) u\left(z^{\prime}\right) d z^{\prime} .
\end{aligned}
$$

Représentant $u$ par une dérivée de la fonction continue $f$, il vient:

$$
\begin{aligned}
& v(x)=\int p\left(x^{\prime}, x_{n} ; x^{\prime}-z^{\prime}, x_{n}\right)\left(\frac{\partial}{\partial z^{\prime}}\right)^{a^{\prime}} f\left(z^{\prime}\right) d z^{\prime} \\
& v(x)=\int\left(\frac{\partial}{\partial x^{\prime}}\right)^{\alpha^{\prime}} p\left(x^{\prime}, x_{n} ; x^{\prime}-z^{\prime}, x_{n}\right) f\left(z^{\prime}\right) d z^{\prime}
\end{aligned}
$$

Cette expression et la caractérisation ([13]) du noyau de $p$ montrent que pour $l$ suffisamment grand, $x_{n}^{l} v(x)$ est une fonction continue.

\section{§ 3. - Existence de valeurs au bord transrerses d'ordre queleonque pour toute solution de l'equation homogene.}

Nous indiquons deux raisonnements permettant de prouver l'existence de valeurs au bord transverses d'ordre quelconque pour une solution $u$ de l'opérateur différentiel elliptique $P$ defini au voisinage de la variété à bord $\Omega$ (le bord $\omega$ est supposé très régulier). Le premier raisonnement (proposition (26)) est une variante d'un raisonnement de HöRMANDER ([10]). 
Le deuxième raisonnement suppose que l'opérateur $P$ ne comporte pas de terme rectangle, mais il ne suppose pas que $P$ est un opérateur elliptique. On indique ensuite des corollaires de l'existence de ces valeurs au bord: d'abord une extension de la formule de GreEN (proposition (29)) puis des conditions suffisantes pour qu' une solution de l'équation homogène en dehors d'une variété régulière soit en fait une solution $C^{\infty}$ définie dans tout l'espace.

\section{Proposition.}

Soit $P$ un opérateur différentiel elliptique défini sur $M$ au voisinage de la variéte à bord $\mathrm{Q}(\mathrm{Q}$ et $\boldsymbol{M}$ de même dimension $n$, le bord $\omega$ est supposé très régulier, mais $\mathbf{\Omega}$ n'est pas forcément compact). Soit $u$ dans $\mathfrak{D}_{P}^{\prime}(\mathbf{\Omega})$ tel que $P u=0$ dans $\mathbf{Q}$. Alors $u$ appartient $\dot{a} \mathfrak{C}^{\infty}\left(\boldsymbol{R}_{x_{n}}^{+}, \mathfrak{D}^{\prime}\left(\boldsymbol{R}_{x^{\prime}}^{n-1}\right)\right)$, ce qui signifie que pour que pour tout $\mu, u$ admet une valeur au bord transverse d'ordre $\mu$ (définition (9)).

Preuve.

On utilise les notations de $\S \S 1$ et 2 . Soit $T$ un paramétrix compact (compactly supported dans la terminologie de [9]). Il suffit done de montrer vu (9), que pour tout $v$,

$$
\left(D_{n}^{\mu} T\left(u \otimes \delta^{(v)}\right)\right)\left(x, x_{n}\right) \quad \text { avec } \quad u \in \mathcal{G}^{\prime}\left(\boldsymbol{R}_{x^{\prime}}^{n-1}\right)
$$

tend vers une limite dans $\mathfrak{D}^{\prime}\left(\boldsymbol{R}^{n-1}\right)$ lorsque $x_{n}$ tend vers $0\left(x_{n}>0\right)$.

On utilise les résultats de [10]. Si

On pose

$$
t(x, \zeta) \sim \Sigma t_{j}(x, \zeta)
$$

$$
R_{J}(x, \zeta)=\left\{\begin{array}{l}
t(x, \zeta) \text { si }|\zeta| \leq 1 \\
t(x, \zeta)-\sum_{j<J} t_{j}(x, \zeta) \quad \text { si }|\zeta|>1
\end{array}\right.
$$

$\Gamma_{\zeta}$, désigne une courbe simple du demi-plan où $\operatorname{Im} \zeta_{n} \geq 0, \Gamma_{\zeta}$, enlace les poles de $t_{j}\left(x, \zeta^{\prime}, \zeta_{n}\right.$; et si $\left|\zeta^{\prime}\right|<1, \Gamma_{\zeta}$, contient le segment d'extrémités $\pm\left(1-|\zeta|^{2}\right)^{1 / 2}$. Lorsque $u$ est dans $\mathcal{C}_{0}^{\infty}\left(\boldsymbol{R}_{x^{\prime}}^{n-1}\right)$, Hörmander montre que si $x_{n}>0$ :

$$
\begin{aligned}
& D_{n}^{u} T\left(u \otimes \delta^{(v)}\right)=(2 \pi)^{-n} \sum_{j<J} \int e^{i<x^{\prime}, \psi^{\prime}>} \hat{u}\left(\zeta^{\prime}\right) d \zeta^{\prime} \int_{\Gamma \xi^{\prime}}\left(( D _ { n } + \zeta _ { n } ) \mu t _ { j } \left(x, \zeta \zeta_{n}^{\mu} x e^{i} x \eta_{n} n d \zeta^{n}\right.\right. \\
& +(2 \pi)^{-n} \int\left(\left(D_{n}+\zeta_{n}\right)^{\mu} R_{J}(x, \zeta)\right) \zeta_{n}^{\gamma} \hat{u}\left(\zeta^{\prime}\right) e^{i<x, \zeta>} d \zeta^{\prime}=\sum_{j<J} I_{j}+\Pi
\end{aligned}
$$

$D_{n}^{\mu} T\left(u \otimes \delta^{\nu}\right)$ apparait donc comme la somme de deux termes que l'on note $\sum_{j<J}^{n} I_{j}$ et II. Lorsque $u$ est une distribution à support compact, â $\left(\zeta^{\prime}\right)$ est une 
fonction à croissance lente. Donc pour $u$ dans $\mathscr{G}^{\prime}$, II est une fonction continue de $\left(x^{\prime}, x_{n}\right)$ si $J$ est suffisamment grand; ce qui prouve que la partie de $D_{n}^{\mu}\left(T\left(u \otimes \delta^{2}\right)\right)\left(\cdot, x_{n}\right)$ qui correspond à II tend vers une limite dans $\mathscr{D}^{\prime}\left(\boldsymbol{R}_{x^{\prime}}^{n-1}\right)$ lorsque $x_{n}$ tend vers 0 . Il nous suffit de considerer la partie qui correspond à $\sum_{j J} I_{j}$. Soit $\varphi$ dans $\mathcal{C}_{0}\left(\boldsymbol{R}_{x^{\prime}}^{n-1}\right.$. Il nous suffit de montrer $q u e$

$$
\left(I_{j}, \varphi\right)=(2 \pi)^{-n} \iint \varphi\left(x^{\prime}\right) e^{i<x^{\prime}, \vartheta^{\prime}>\hat{u}\left(\zeta^{\prime}\right) d x^{\prime} d \zeta^{\prime}} \int_{\zeta_{\zeta^{\prime}}}\left(( D _ { n } + \zeta _ { n } ) \mu t _ { j } \left(x, \zeta \zeta_{n}^{\mu} e^{i x_{n} \xi_{n}} d \zeta_{n}\right.\right.
$$

est défini pour $u$ dans $\mathcal{O}^{\prime}$ et tend vers une limite lorsque $x_{n}$ tend vers 0 .

Or $\left(I_{j}, \varphi\right)=\int \hat{u}\left(\zeta^{\prime}\right) A_{j} d \zeta^{\prime}$ avec

$$
A_{j}=\int \varphi\left(x^{\prime}\right) e^{i<x^{\prime}, \zeta^{\prime}>}\left[\int _ { \zeta _ { \zeta ^ { \prime } } } \left(\left(D_{n}+\zeta_{n}\right)^{\mu} t_{j}\left(x, \zeta \zeta_{n}^{\mu} e^{i x_{n}^{2} n} d \zeta_{n}\right] d x^{\prime}\right.\right.
$$

et une intégration par parties donne

$$
\zeta^{\prime \alpha} A_{j}=\int e^{i\left\langle x^{\prime} ; \zeta^{\prime}\right\rangle}\left(\frac{1}{i} \frac{\partial}{\partial x^{\prime}}\right)^{\alpha}\left[\varphi ( x ^ { \prime } ) \int \left(\left(D_{n}+\zeta_{n}\right)^{\mu} t_{j}\left(x, \zeta \zeta_{n}^{\mu} e^{i x_{n} \zeta_{n}} d \zeta_{n}\right] d x^{\prime}\right.\right.
$$

Comme le crochet est uniformément borné lorsque $x_{n}$ tend vers 0 par $C\left|\zeta^{\prime}\right|^{v+\mu+s_{j}+1}$, il en résulte que $\left\langle I_{j}, \varphi\right\rangle$ est défini pour $u$ dans $\mathcal{G}^{\prime}$ et tend vers une limite lorsque $x_{n}$ tend vers 0 .

$$
\text { proposition. }
$$

Soit $P$ un opérateur différentiel hypoelliptique sur $\boldsymbol{K}_{x}^{n}$, ̀̀ coefficients $\mathfrak{C}^{\infty}$ et s'écrivant

$$
P(x, D)=P_{0}^{\prime}\left(x, D^{\prime}\right)+a(x) D_{n}^{n}
$$

avec $m>0$ et $a(x)+0 . P_{0}^{\prime}$ ne fait intervenir que $D^{\prime}$ (les dérivations tangentielles) Soit $u$ définie pour $x_{n}>0$ telle que la fonetion $u_{0}$ égale à $u$ pour $x_{n}>0$ et à 0 pour $x_{n}<0$ soit localement intégrable dans $\boldsymbol{R}_{n}$. On suppose que $P u=0$ (pour $\left.x_{n}>0\right)$. Alors pour $\mu(0 \leq \mu \leq m-1)$.

$$
\left(\frac{1}{i} \frac{\partial}{\partial x_{n}}\right)^{\mu} u(\cdot, \varepsilon)=\gamma_{\mu}^{\varepsilon} u
$$

tend vers une limile dans $\mathscr{D}^{\prime}\left(\boldsymbol{R}_{x^{\prime}}^{n-1}\right)$ lorsque $\varepsilon \downarrow 0$.

Preuve.

Soit $\varphi$ dans $\mathcal{C}_{0}^{\infty}\left(\boldsymbol{R}_{x^{\prime}}^{n-1}\right)$ nulle pour $\left|x^{\prime}\right| \geq A$ et $\psi$ dans $\mathcal{C}_{0}^{\infty}\left(\boldsymbol{R}_{x_{n}}\right)$ valant 1 au voisinage de l'origine. On applique la formule de GRTEN (15)' à $P$, au cylindre 
$C$ où $\varepsilon_{1}<x_{n}<\varepsilon_{2}$ et $\left|x^{\prime}\right| \leq 2 A$. En égalant les actions des 2 distributions figurant dans les 2 membres de $(15)^{\prime}$ sur la fonction test $\varphi \psi$, on obtient:

$$
\iint_{C} u^{t} P(\varphi \psi)-0=\int_{\partial C}(Q \gamma u) \varphi \psi
$$

Au premier membre, tout terme correspondant à un opérateur de dérivation normal dans ' $P$ est nul. Le premier membre se réduit done à:

$$
\iint_{C} u^{\imath} P_{0} \varphi
$$

De même, au deuxième membre, tonte multicouche (d'ordre $\geq 1)$ de $Q(\gamma u)$ donne une contribution nulle. Vue la formule (2.2.1) de [9], le $2^{e}$ membre se réduit à :

$$
i^{-1} \sum_{l=0}^{m-1} \int_{\partial C}\left(P_{l+1}\left(x, D^{\prime}\right) u_{l} \delta\right) \varphi \psi=i^{-l} \int_{\partial C} a(x) u_{m-1}(x) \varphi\left(x^{\prime}\right) d x^{\prime}
$$

D' où

$$
\int_{x_{n}=\varepsilon_{2}} a(x) u_{m-1}(x) \varphi\left(x^{\prime}\right) d x^{\prime}-\int_{x_{n}=\varepsilon_{2}} a(x) u_{m-1}(x) \varphi\left(x^{\prime}\right) d x^{\prime}=i \iint_{C} u(x)\left({ }^{\imath} P_{0} \varphi\right)\left(x^{\prime}\right) d x
$$

Si $u$ est localement intégrable, le $2^{e}$ membre tend vers 0 lorsque $\varepsilon_{1}$ et $\varepsilon_{2}$ tendent vers 0 . La dernière relation montre alors que $\left(a u_{m-1}\right)\left(\cdot, x_{n}\right)$, done $u_{n-1}\left(\cdot, x_{n}\right)$ tendent vers des limites dans $\mathfrak{D}^{\prime}\left(\boldsymbol{R}_{x^{\prime}}^{n-1}\right)$ lorsque $x_{n}$ tend vers 0.

Done $u_{m-1}$ appartient à $\mathfrak{C}^{0}\left(\boldsymbol{R}_{x_{n}}^{+}, \mathfrak{D}^{\prime}\right)$. Il en résulte que les primitives (par rapport à $x_{n}$ ) de la fonction vectorielle $u_{m-1}$ appartiennent aussi à $\mathfrak{C}^{0}\left(\boldsymbol{R}_{x_{n}}^{+}, \mathfrak{D}^{\prime}\right)$, donc que $u_{m-1}$ est $(m-1)$ fois dérivable à valeurs dans $\mathfrak{D}^{\prime}$.

\section{Corollaires.}

a) Généralisation des classes $H^{P}$ de Hardy.

$u^{0}$ est localement sommable en particulier si

$$
u \in L^{\infty}\left(\boldsymbol{R}_{x_{n}^{+}}^{+}, L^{p}\left(\boldsymbol{R}_{x^{\prime}}^{n-1}\right)\right) \text { avec } 1<p<\infty .
$$

Nous dirons que les solntions $u$ de $P u=0$ vérifiant cette condition appartiennent à la classe $H^{p}$ relative à $P$. Vue (27), pour $0 \leq \mu \leq m-1$, $\gamma_{\mu}^{\varepsilon} u$ tend vers une limite dans $\mathfrak{D}^{\prime}$. En particulier, les $\gamma_{0}^{\varepsilon} u$ décrivent un borné de $L^{P}$ et convergent dans $\mathfrak{D}^{\prime}$. Comme la bouble unité de $L^{P}$ est faiblement fermée dans $\mathfrak{D}^{\prime}$, on voit que $u$ admet ne valeurs au bord dans $L^{P}$. 
b) Valeurs au bord d'une fonction $f(z)$ holomorphe pour $y=\operatorname{Im} z>0$ et à croissance lente au voisinage de l'axe réel $x^{\prime} 0 x$. Il est bien connu qu' une telle valeurs au bord existe dans $\mathfrak{D}^{\prime}$. On pent retrouver ce résultat en appliquant (27) à l'opérateur $P=\frac{\partial}{\partial z}$ et à une primitive complexe de $f$ d'ordre
convenable (exercice).

\section{Proposition (formule de Green)}

Soit $u$ dans $\mathfrak{D}_{P}^{\prime}(\Omega)$ avec $P u=0$ dans $\Omega . \gamma u=\left(\gamma^{0} u, \ldots \gamma_{m-1} u\right)$ désigne l'ensemble des valeurs au bord transverses $\gamma_{j} u$ avec $0 \leq j \leq m-1$. Soit $u^{0}$ le prolongement canonique de $u$.

Alors

$$
P\left(u^{0}\right)=\tilde{P}(\gamma u)
$$

En effet.

Soit $\Omega_{\varepsilon}$ la variété à bord limitée par $\omega_{\varepsilon}, \boldsymbol{u}^{\varepsilon}$ désigne la distribution sur $M$ représentée par la fonction égale à $u$ sur $\boldsymbol{Q}_{\varepsilon}$ et par 0 dans le complémentaire de $\Omega_{\varepsilon}$. Lorsque $\varepsilon$ tend vers $0, u^{\varepsilon}$ tend vers une distribution $u^{0}$ sur $M$, appelée le prolongement cañonique de $u$. La formule de GreEN appliquée à $\Omega_{\varepsilon}$ donne

$$
P\left(u^{\S}\right)=\tilde{P}\left(\gamma^{\hat{\imath}} u\right)
$$

avec $\gamma^{\varepsilon} u=\left(\gamma_{\theta}^{\varepsilon} u, \ldots \gamma_{m-1}^{\varepsilon} u\right)$.

Lorsque $\varepsilon$ tend vers 0, va $(26), \gamma_{j}^{\varepsilon} u$ tend vers une limite notée $\gamma_{j} u$. On a donc prouvé (30).

\section{Corollaire (principe du prolongement hypoelliptique).}

On suppose $P$ hypoelliptique. On pose $\mathbf{\Omega}^{0}=\Omega$ et $\mathbf{\Omega}^{1}=M \backslash \Omega$. On se donne $u^{j}$ dans $\mathfrak{D}_{P}^{\prime}\left(\mathrm{Q}^{j}\right)$ avec $P u^{j}=0$ sur $\Omega^{j}, j=0$ on 1 . On suppose que $u^{0}$ et $u^{1}$ admettent les mêmes valeurs au bord jusqu' à l'ordre $m-1$. Alors le conple $\left(u^{0}, u^{1}\right)$ définit une fonction $\mathcal{C}^{\infty}$ sur $M$.

En effet, notons $\tilde{u^{0}}$ et $\tilde{u}^{1}$ les prolongements canoniques de $\boldsymbol{u}^{0}$ et $\boldsymbol{u}^{1}$. Les formules de GREEN relatives à $\Omega^{0}$ et $\Omega^{1}$ s' écrivent $P\left(\tilde{u}^{0}\right)=\tilde{P}^{0}(\gamma u)$ et $P\left(\tilde{u}^{1}\right)=\tilde{P}^{1}(u)$. Comme les second membres représentent des distributions de signes contraires, on a

$$
P\left(\tilde{u}^{0}+\tilde{u}^{1}\right)=0 .
$$

La conclusion en résulte puisque $P$ est hypoelliptique. 


\section{$\S$ 4. - Deux Interpretations du probleme (1)}

Considérons le problème abstrait (1). Nous allons considérer deux variantes de ce problème.

\section{Interpretation 1.}

$u$ est dans $\mathfrak{C}^{k}\left(\boldsymbol{R}_{x_{n}}, \mathfrak{D}^{\prime}\left(\boldsymbol{R}_{x^{\prime}}\right)\right)$.

Pour tout $\mu$ tel que $0 \leq \mu \leq k,\left(\frac{1}{i} \frac{\partial}{\partial x_{x}}\right)^{\mu} u$ définit une fonction continue de la variable $\boldsymbol{x}_{n}$, ̀̀ valeurs dans $\mathscr{D}\left(\boldsymbol{R}_{x^{\prime}}^{n-1}\right)$ et par conséquent $\gamma_{\gamma} u$ pent être interprété dans (1) comme une valeur au bord.

\section{Interprétation 2.}

$u$ est dans un sous espace topologique $X$ de $\mathfrak{D}_{P}^{\prime}(\Omega)$ :

$X$ étant tel que $\mathcal{C}^{\infty}(\bar{\Omega})$ est dense dans $X$ et ponr tout $\mu(0 \leq \mu \leq k)$, l'application

$$
\begin{aligned}
\mathcal{C}^{\infty}(\overline{\mathbf{\Omega}}) & \rightarrow \mathcal{C}^{\infty}(\omega) \\
\varphi & \rightarrow\left(\frac{1}{i} \frac{\partial}{\partial \boldsymbol{x}_{n}}\right)_{\varphi}^{\mu}
\end{aligned}
$$

se prolonge en une application continue de $X$ dans $\mathfrak{D}^{\prime}(\omega)$. Dans ces conditions $\gamma_{\mu} u$ doit être interprété dans (1) comme une trace.

Espace $\tilde{X}$.

(32) Etant donné un tel espace $X$, on appelle ci-après $\tilde{X}$ tout sous espace vectoriel topologique de $\mathscr{E}^{\prime}(M)$ tel que l'application naturelle de restriction

$$
\text { rest: } \quad \tilde{X} \rightarrow X
$$

admette un relèvement continu.

$$
\text { rel : } \tilde{X}-X \text { avec restr o rel }=\mathrm{Id} \text {. }
$$

Notons que les interprétations 1 et 2 du problème (1) ne sont pas toujours équivalentes. L'interprétation 2 est plus courante que l'interprétation 1 ; et il existe des cas où l'interprétation 1 est strictement plus forte que l'interprétation 2: e'est par exemple le cas des expaces $H^{s}$ avec poids.

\section{Proposition}

On considére le problème (1), avec $f$ dans $\mathfrak{D}^{\prime}(\Omega, F)$ et $g$ dans $\mathfrak{D}^{\prime}(\omega, G)$. On cherche $u$ dans $\mathfrak{D}_{P}^{\prime}(\mathbf{Q}, E)$ 
a) Pour que ce problème ait un sens, $\gamma_{\mu} u(1 \leq \mu \leq k)$ étant la valeur au bord de $\left(\frac{1}{i} \frac{\partial}{\partial x_{n}}\right)^{\mu} u$, il faut et il suffit que $f$ admette un prolongement $\tilde{f}$ tel que

$$
T \tilde{f} \in \mathfrak{C}^{\bar{k}}\left(\boldsymbol{R}_{x_{n}}^{+}, \quad \mathfrak{D}^{\prime}\left(\boldsymbol{R}_{x^{\prime}}^{n-1}\right)\right)
$$

b) Pour que ce problème ait un sens, $\gamma_{\mu} u(1 \leq \mu \leq k)$ étant la $X-$ trace $d^{\prime}$ ordre $\mu$ de la distribution $u$ de $X(X$ étant un sous espace vectoriel topologique quelconque de $\mathscr{D}_{P}^{\prime}(\overline{\mathcal{Q}}), \mathcal{C}^{\infty}(\overline{\Omega)}$ étant dense dans $X)$ il faut et il suffit que $f$ admette un prolongement $\tilde{f}, T \tilde{f}$ admettant des $\tilde{X}$ - traces fonctionnelles jusqu' $\grave{a}$ $l$ 'ordre $k$.

\section{Démonstration.}

Comme $P$ envoie $\mathscr{D}_{P}^{\prime}(\Omega)$ dans $\mathfrak{D}_{P}^{\prime}(\Omega)$ il faut supposer que $f$ est dans $\mathfrak{D}_{P}(\Omega)$ mais ceci ne suffit pas, pour assurer l'existence de traces ou de valeur au bord pour $u$ et $D_{n}^{\mu} u$.

a) Soit $\tilde{u}$ une distribution sur $M$ prolongeant $u$. Alors $\tilde{f}=P u$ est un prolongement de $f$. Il existe un opérateur régularisant $r$ tel que

$$
T P \tilde{u}=u+\tilde{u}=\tilde{T f}
$$

Or:

(35) $\tilde{u}$ admet des valeurs au bord transverses jusqu'à l'ordre $k$ si et seulement si, il en est ainsi de $u$

(36) $\quad r \tilde{u}$ admet des valeurs au bord transverses d'ordre quelconque.

De $(34),(35)$ et $(36)$, il résulte que $u$ admet des valeurs au bord transverses jusqu'à l'ordre $k$, si et seulement si, il en est ainsi de $T \tilde{f}$.

b) On se demande quand il existe un sous espace $X$ de $\mathscr{D}_{P}^{\prime}(\Omega)$ tel que $u$ ait des $X$ - traces normales jusqu'à l'ordre $k$. Soit $\tilde{X}$ défini par (32). Or

(35) $\tilde{u}$ admet des $\tilde{X}$ - traces transverses jusqu'à l'ordre $k$ si et seulement si $u$ admet des $X$ - traces transverses jusqu' à l'ordre $k$.

En effet si $\tilde{u}$ admet des $\tilde{X}$ - traces jusqu'à l'ordre $k$, alors $u$ admet des $X$ - traces car l'application $\gamma_{\mu}$ o rel:

$$
X \stackrel{r e l}{\rightarrow} \tilde{X} \stackrel{\Upsilon_{\mu}}{\rightarrow} \mathscr{D}^{\prime}(\omega)
$$

est continue pour $1 \leq \mu \leq k$. La réciproque se montre de même car $\gamma_{\mu}$ o restr est continue 
$(36)^{\prime} \quad r \tilde{u}$ admet toujours des $\tilde{X}$ - traces normales jusqu'à l'ordre $k$. En effet. l'application $\gamma_{\mu} r$ est continue

$$
\tilde{X} \stackrel{r}{\rightarrow} \mathcal{C}^{\infty}(M) \stackrel{r \mu}{\longrightarrow} \mathbb{C}^{\infty}(\omega) \subseteq \mathfrak{D}^{\prime}(\omega)
$$

De $(3 t),(35)^{\prime}$ et $(36)^{\prime}$ il résulte que $u$ admet des $X$-traces normales jusqu'à l'ordre $k$ si et seulement si $T \tilde{f}$ admet des $\tilde{X}$-traces normales jusqu'à l'ordre $k$.

(37) Exemple d'application de la proposition (33).

Supposons que $t$ soit dans $H^{s}(\boldsymbol{Q})$ et cherchons pour quelles valeurs de $s$ (réelles par exemple) le problème (1) a un sens. On sait qu'il existe $\tilde{f}$ dans $H^{s}|M|$ qui prolonge $f$. Pour que $u$ admette des valeurs an bord normales (resp des $H^{s}$ - traces normales) jusqu'à l'ordre $k$, il faut et il suffit qa'il en soit de même de $T \tilde{f}$. Or $T$ etant un o.p.d. d'ordre - $-m$, $T \tilde{f}$ appartient is $H^{s+m}(M)$.

Done $T \tilde{f}$ admet des valeurs au bord normales (resp des $H^{s}$ - traces normales) jusqu' à l'ordre $k$ si et seulement si $m+s-k>\frac{1}{2}$; soit $s>k-m+\frac{1}{2}$. Done lorsque $f$ décrit $H^{s}(\Omega)$, le problème (1) a un sens pour l'interprótation 1 (resp 2) si et seulement si $s>k-m+\frac{1}{2}$ (Lions et Magenis ont prouvé dans le cas où $P$ est une équation et où $B$ est un système normal qui recouvre $P$, que la condition $s>-\frac{1}{2}$ est suffisante; voir [14], page 204).

\section{§ 5. - Resolution et discussion du probleme (1).}

Nous supposons à présent que $f$ appartient au sous-espace $H$ de $\mathfrak{D}_{P}^{\prime}(\Omega, F)$ des distributions $f$ qui vérifient les conditions de (33). Pour résoudre et discuter le problème (1), il nous suffit de vérifier que les raisonnements de L. HönMANDER ([10]) se transposent à notre cas, ce que nous faisons brièvement ci-après. On suppose que $P T=I d, T P=I d$ pour simplifier l'exposé (voir rédaction détaillée dans [13]). $\tilde{f}$ étant un certain prolongement de $f$, en remplaçant $u$ par $u-T \tilde{f}$, on se ramène au cas où $f=0$.

\section{Proposition.}

a) Si le problème (1) est elliptique à droite, alors il existe des solutions de (1) sauf si $f, g)$ appartient à un sous espace de $\omega$ dimension finie de $H \times \mathscr{D}^{\prime}(\omega)$.

b) Si le problème (1) est elliptique à gauche, alors le système homogène associé à (1) admet au plus un nombre funi de solutions u linéairement indé- 
pendantes et la régularité des solutions est liée à la régularité des données par les relations (34), (39) et (40)

Preuve.

On appelle $Q$ le «projecteur sur les bonnes données de CAUCHY ([4], [5], [10] et [14])

$$
Q(u)=\gamma T \tilde{P}_{\gamma_{u}}
$$

$B Q$ est un o.p.d: $\mathfrak{C}^{\infty}\left(\omega, E^{m}\right) \rightarrow \mathcal{C}^{\infty}(\omega, G)$

a) Pour prouver l'existence d'une solution de (1), on suppose que le problème est elliptique à droitè, c'est-à-dire que $B Q$ a un symbole principal surjectif. Alors, vu [2], $B Q$ a un quasi-inverse à droite:

$$
\begin{gathered}
R: \quad \mathfrak{C}^{\infty}(\omega, G) \rightarrow \mathfrak{C}^{\infty}\left(\omega, E^{m}\right) \\
B Q R=I+r
\end{gathered}
$$

Cherchant une solution $\gamma u$ sous la forme $\gamma u=R v$

$$
\text { d'oì } v+r v=g
$$

$v$ est la solution d'une équation de Fredholm.

b) Pour étudier la dimension du noyau et la régularité de la solution en fonction de la régularité des données, on remarque que

$$
\begin{gathered}
(Q-i d) \curlyvee u=0 \\
B \gamma u=g
\end{gathered}
$$

(On suppose que le problème est elliptique à gauche, éest-à-dire que l'o.p.d: (id $-Q) \oplus B$ a un symbole injectif. Alors, vu [2], cet o.p.d. admet un quasi-inverse à gauche $L$ :

$$
L[(i d-Q) \oplus B]=i d+r^{\prime}
$$

(40) D'où $L(0, g)=\gamma u+r^{\prime} \gamma u$, ce qui proure que $\gamma u$ est régulier si g l'est. 


\section{BIBLIOGRAPHIE}

[1] Agmon Douglis et L. Nirenberg, Comm. Pure Appl. Math. 12, (1959) pp. 623-727.

[2] AттуAн et R. Bотt, Lefschets fixed point theorem, Annal of Mathematics. Princeton (1967).

[3] L. Boutet de Monvel, Comportement d'un o.p.d. sur une variété à bord Ir, Journ. d'Anal Math Jerusalem XVII (1966) pp. 20̃õ.304.

[4] - - Thèse-à paraìtre.

[5] A.P. Oalderon, Communieation au Symposium de Novosibirsk. (1963).

[6] P. Grisvard, Espaces intermédiaires entre espaces de Sobotev avec poids, Annali della seuola Norm. Sup. di Pisa. série III XVII fase. 3 (1963) pp. $20 \%$ \%̆-296.

[7] L. Hörmander, Linear differential operators, Springer, Berlin, 1963.

[8] - -, Psendo differential operators, Comm. Pure Appl. Math. 18 (1965) pp. 501.517.

[9] - -, Pseudo differential operators and hypoelliptic equations, Proceeding of symposia in Pure Mathematies (AMS, $\mathrm{n}^{0}$ 10, 1967). pp. 138.183.

[10] - - Pseudo differential operators and no elliptic boundary, problems, Ann. of Math. 83 (1966), pp. 129.209.

[11] - - Séminaire à Princeton (1986).

[12] - - Cours à Stanford University (Juillet 1967).

[13] P. Krée, Cours à Bari (mai 1968), Pubblicazione di l'Instituto di Analysa Mathematica (à paraître).

[14] J. L. Lrons et E. Magenés. Problémes aux limites non homogènes et applications, vol. 1, Dunod, Paris, 1968.

[15] J. Pewrne, Another approach to the theory of elliptic boundary value problems, Comm. Pure Appl. Math. 14 (1951) pp. 711-731.

[16] R. T. SemLwy, Singular integrals and boundary problems, Aemr. Journal of Math (1966).

[17] L. Schwartr, Theorie des distributions, tome 1, Hermann. Paris 1951.

[18] — - Distributions semi-régulières et changements de coordonnées, J. Math. pures et applications. $9 e$ série, t. 36 (1957), pp. 109.127.

[19] M. VIsıK et Eskin, Equations intégrales singulières sur une variété à bord, Uspehi mat. Nauk, XV. 3, 123, (1965) pp. 89.152.

[20] E. M. Stein el G. Weiss, Act. Math. 103, (1960) pp. 25.62. 